\title{
The Influence of Constipation on Asthma: A Real-world, Population-based Cohort Study
}

\author{
Yen Chu Huang ${ }^{1}$, Meng Che Wu ${ }^{1}$, Yu-Hsun Wang ${ }^{2}$, and James Cheng-Chung $\mathrm{Wei}^{3}$ \\ ${ }^{1}$ Taichung Veterans General Hospital \\ ${ }^{2}$ Chung Shan Medical University, Taichung, \\ ${ }^{3}$ Chung Shan Medical University
}

January 17, 2021

\begin{abstract}
Background Among respiratory diseases, asthma is one of the most burdensome disorder worldwide. Growing evidence disclose gut dysbiosis may contribute to asthma via the gut-lung axis. Constipation can lead to alteration of the gut microflora. The clinical impact of constipation on asthma has not been researched. Therefore, we aim to assess the risk of asthma in constipated patients by a nationwide population-based cohort study. Methods We analyzed 82421 constipated patients and 82421 individuals without constipation between 1999 and 2013 from the Taiwanese National Health Insurance Research Database. Analysis of propensity score was utilized to match age, gender, comorbidities, and medications at a ratio of 1:1. Besides, multiple Cox regression analysis was performed to evaluate the adjusted hazard ratio of asthma. Furthermore, sensitivity tests and a stratified analysis were conducted. Results The incidence of asthma was 10.8 per 1,000 person-years in the constipation group, which was higher than the rate of 5.6 per 1,000 person-years observed in the non-constipation group. After adjustment for age, gender, comorbidities, and medications, constipated patients had a 1.91-fold greater risk of asthma compared to those without constipation (adjusted hazard ratio [aHR]: 1.91 (95\% C.I. 1.84-1.99). In subgroup analyses, patients aged 20-39 years had a 2.04-fold highest risk of asthma in the constipation cohort (aHR:2.04, 95\% CI, 1.84-2.26). Besides, the severity of constipation is associated with an increased risk of asthma; the aHR was $1.76(1.69-1.85), 2.15(2.03-2.27)$, and 2.29(2.10-2.49) for $<3$ times, 3-12 times, and [?]12 times of laxatives prescription within one year, respectively. $(\mathrm{p}<0.001)$ Moreover, constipated patients had a higher likelihood of asthma, regardless of gender, comorbidities, and medications. Conclusion Constipation relates to a significantly increased risk of asthma. Physicians should be aware of the possibility of asthma in constipated people. Further research is warranted to investigate the possible pathological mechanisms of this association.
\end{abstract}

\section{The Influence of Constipation on Asthma: A Real-world, Population-based Cohort Study} Yen-Chu HUANG, ${ }^{1,++}$ Meng-Che WU, ${ }^{1},++$, Yu-Hsun WANG, ${ }^{2}$ James Cheng-Chung WEI ${ }^{3,4}$

${ }^{1}$ Division of Pediatric Gastroenterology, Children's Medical Center, Taichung Veterans General Hospital, ${ }^{2}$ Department of Medical Research, Chung Shan Medical University Hospital,

${ }^{3}$ Division of Allergy, Immunology and Rheumatology, Chung Shan Medical University Hospital; Institute of Medicine, College of Medicine, Chung Shan Medical University, ${ }^{4}$ Graduate Institute of Integrated Medicine, China Medical University

\section{Short running title: Constipation and asthma}

Correspondence: James Cheng-Chung Wei, Institute of Medicine, Chung Shan Medical University, No. 110, Sec 1, Jianguo N. Road, Taichung City, 40201, Taiwan. Email: jccwei@gmail.com

Disclosures relevant to this paper: None 
++ Yen-Chu Huang and Meng-Che Wu are co-first authors

\section{ABSTRACT \\ Background}

Among respiratory diseases, asthma is one of the most burdensome disorder worldwide. Growing evidence disclose gut dysbiosis may contribute to asthma via the gut-lung axis. Constipation can lead to alteration of the gut microflora. The clinical impact of constipation on asthma has not been researched. Therefore, we aim to assess the risk of asthma in constipated patients by a nationwide population-based cohort study.

\section{Methods}

We analyzed 82421 constipated patients and 82421 individuals without constipation between 1999 and 2013 from the Taiwanese National Health Insurance Research Database. Analysis of propensity score was utilized to match age, gender, comorbidities, and medications at a ratio of 1:1. Besides, multiple Cox regression analysis was performed to evaluate the adjusted hazard ratio of asthma. Furthermore, sensitivity tests and a stratified analysis were conducted.

\section{Results}

The incidence of asthma was 10.8 per 1,000 person-years in the constipation group, which was higher than the rate of 5.6 per 1,000 person-years observed in the non-constipation group. After adjustment for age, gender, comorbidities, and medications, constipated patients had a 1.91-fold greater risk of asthma compared to those without constipation (adjusted hazard ratio [aHR]: 1.91 (95\% C.I. 1.84-1.99). In subgroup analyses, patients aged 20-39 years had a 2.04-fold highest risk of asthma in the constipation cohort (aHR:2.04, 95\% CI, 1.84-2.26). Besides, the severity of constipation is associated with an increased risk of asthma; the aHR was 1.76 (1.69-1.85), 2.15(2.03-2.27), and 2.29(2.10-2.49) for $<3$ times, 3-12 times, and [?]12 times of laxatives prescription within one year, respectively. $(\mathrm{p}<0.001)$ Moreover, constipated patients had a higher likelihood of asthma, regardless of gender, comorbidities, and medications.

\section{Conclusion}

Constipation relates to a significantly increased risk of asthma. Physicians should be aware of the possibility of asthma in constipated people. Further research is warranted to investigate the possible pathological mechanisms of this association.

Key words: constipation, asthma, Longitudinal Health Insurance Database, gut microflora, dysbiosis

\section{What's Known?}

Recent studies showed that intestinal dysbiosis contributes to asthma via the gut-lung axis.

Constipation can lead to alteration of the gut microflora.

\section{What's New?}

Constipated people had a 1.91-fold greater risk for asthma compared with those without constipation. Furthermore, patients with constipation had a higher likelihood of asthma, regardless of gender, comorbidities, as well as the usage of corticosteroids and antihistamines.

Constipation relates to a significantly risk factor of asthma. Physician should take care of the possibility of developing asthma in constipated patients.

\section{INTRODUCTION}

Among respiratory diseases, asthma is one of the most burdensome disorder both affects in childhood and adulthood. The prevalence was estimated that there were more than 272 million people had asthma worldwide in 2017.[1] Asthma not only increases financial, social, and psychological burdens, but also makes a 
harmful impact on quality of life, and is connected with comorbidities.[2] Interestingly, there is increasing evidence presenting an connection between asthma and constipation[3], a common condition affecting adults and children globally and a frequent reason for visits to physicians. The mean global prevalence of constipation in children is $12 \%$ and that in adult is $16 \%$. [4] Even though constipation has few complications of life-threatening, it can make physical and mental distress for patients and their families, eventually impairing quality of life. Complications of constipation included hemorrhoids, rectal prolapse, and fecal or urine incontinence, which usually increases the frequency of hospitalizations or outpatient department visits, leading to increased burdens of health insurance.

Nowadays, constipation is seemed to be a causative factor in intestinal dysbiosis[5] and therapeutic managements are increasingly incorporating prebiotics, probiotics[6], or synbiotics with a view to regulating the intestinal microflora[7, 8]. Moreover, recent research has demonstrated that the gut microbiota would exert important regulatory effects via the gut-lung axis.[9-11] For instance, gut dysbiosis[12] and lower concentration of short-chain fatty acids (SCFAs) in the bowel are found in patients with asthma. This condition decreases the production of butyrate and propionate, leading to the dysregulation of gut inflammation and the defect of the intestinal epithelial barriers, resulting in leaky gut and allow penetration of microorganisms and toxins into systemic circulation, thereby activating Th2 immune responses, eventually contributing to airway inflammation. Some research have suggested that allergic diseases might be associated with constipation.[13, 14] Furthermore, previous study has demonstrated that stool stasis in the long time could influence the microbiota and environment of gut, resulting in deleterious effects on mucosal immunity and intestinal motility.[15] Whether if constipation predisposes susceptible people to asthma is unknown. At present, there are still scanty data on the connection between constipation and asthma in the literature. In addition, this association has never been investigated using a large-scale national longitudinal database. We hypothesized that constipation might influence the risk of asthma and assessed this hypothesis by analyzing a real-world, population-based retrospective cohort from National Health Insurance Research Database (NHIRD) in Taiwan.

\section{METHODS}

\section{Data source}

This study analyzed the National Health Insurance Research Database (NHIRD) that contains the healthcare data of almost $99 \%$ of Taiwan's entire population, approximately 23 million NHI beneficiaries. The database includes all insurance claims data, including outpatient visits, emergency visits, and hospitalizations. The Longitudinal Health Insurance Database, (LHID) is a subset of the NHIRD comprising one million individuals randomly sampled from the 23 million NHI beneficiaries for the period from 1999 to 2013.[16] The data of patients were de-identified prior to release to the users, in accordance with privacy protocols, and this study was approved by the Institutional Review Board of Chung Shan Medical University Hospital (IRB no. CS15134).

\section{Study group and outcome measurement}

The population was composed of patients with newly diagnosed constipation (ICD-9-CM codes=564.0) from 2000 to 2012. To ensure accuracy of diagnoses, we excluded any diagnosis of constipation before 2000 and only patients with at least three outpatient visits or one hospitalization were selected for inclusion in the final analysis. The index date of this cohort was set as the first date of diagnosis of constipation. Furthermore, to ensure that all individuals had new-onset asthma, we ruled out any diagnosis of asthma (ICD-9-CM=493) happening before the index date. The non-constipation group constituted subjects who had never been diagnosed with constipation for the period 1999 to 2013.

The outcome variable was defined as a diagnosis of asthma with at least three outpatient visits or once hospitalization. The patients were followed up until the occurrence of atopic dermatitis, 31 December 2013, or withdrawal from the National Health Insurance system, whichever occurred first.

\section{Covariates and matching}


The baseline characteristics were age, gender, hypertension (ICD-9-CM=401-405), hyperlipidemia (ICD-9$\mathrm{CM}=272.0-272.4$ ), chronic kidney disease (ICD-9-CM=585), chronic liver disease (ICD-9-CM=571), chronic obstructive pulmonary disease (COPD) (ICD-9-CM =491, 492, 496), cancer (ICD-9-CM=140-208), diabetes (ICD-9-CM=250), cardiovascular disease (ICD-9-CM=410-414), stroke (ICD-9-CM=430-438), and autoimmune disease (ICD-9-CM=710.0, 720.0, 714.0). The comorbidities were defined as occurring within one year prior to the index date with at least three outpatient visits or once hospitalization. Besides, corticosteroids and antihistamines during the study period were included and defined as usage for at least [?]30 days. To assess the severity of constipation, we collected additional information on the total numbers of laxatives prescription (anatomical therapeutic chemical [ATC] classification system code A06A), which occurred within one year after the index date.

Moreover, propensity score matching based on age, gender, hypertension, hyperlipidemia, chronic kidney disease, chronic liver disease, COPD, cancer, diabetes, cardiovascular disease, stroke, autoimmune disease, corticosteroids, and antihistamines was applied to balance the heterogeneity of the two groups. The propensity score was a probability that was estimated through logistic regression. The binary variable was the constipation and non-constipation group.

\section{Statistical analysis}

Comparisons between the constipation group and non-constipation group were using absolute standardized differences (ASD). Whenever the ASD were less than 0.1, the characteristics of the two groups were deemed to be similar. On the other hand, Kaplan-Meier analysis was applied to calculate the cumulative incidence of asthma and then log-rank test was used to test the significance. Cox proportional hazard model was applied to estimate the hazard ratio of asthma between the constipation and non-constipation groups.

\section{RESULTS}

The flowchart is presented in Fig. 1. We identified 82421 patients with constipation and 82421 matched controls between 1999 and 2013 from the LHID. Demographic characteristics of this study participants are shown in Table 1. The constipated patients and non-constipation cohort were similar in age and gender distribution. After propensity score matching, there were no statistically significant differences between the constipation and non-constipation groups.

As shown in Table 2, the incidence of asthma was 10.8 per 1,000 person-years in the constipation group, which was higher than the rate of 5.6 per 1,000 person-years observed in the non-constipation group. After adjustment, patients with constipation had a significantly higher risk of asthma than those without constipation (aHR:1.91, 95\% C.I. 1.84-1.99, $\mathrm{p}<0.001$ ). Compared with women, men had a significantly higher risk of asthma (aHR:1.17; 95\% CI, 1.12-1.22; p<0.001). In term of comorbidities, we observed that people with hypertension, COPD, or autoimmune disease had a relatively higher risk of asthma. (hypertension:1.22, 95\% C.I. 1.15-1.29, $\mathrm{p}<0.001$; COPD:3.21, 95\% C.I. 2.94-3.50, $\mathrm{p}<0.001$; autoimmune disease:1.42, 95\% C.I. 1.17$1.73, \mathrm{p}<0.001)$. By contrast, patients using corticosteroids or antihistamines during the study for period at least 30 days had a lower risk of asthma. (corticosteroids: 0.80, 95\% C.I. 0.76-0.84, p $<0.001$; antihistamines: $0.61,95 \%$ C.I. $0.59-0.64, \mathrm{p}<0.001)$

Table 3 showed that subgroup analyses were performed to assess the association between constipation and asthma based on demographic characteristics. Patients in the constipation group aged 20-39 years, had a 2.04-fold greater risk of asthma compared with the same age group in the non-constipation group (aHR; 95\% CI,1.84-2.26, $\mathrm{P}<0.001)$. Patients aged $<20,40-64$, and [?]65 years in the constipation group had a 1.85, 1.70, and 2.02-fold greater risk of asthma.(aHR; 95\% CI, 1.69-2.01, 1.57-1.83 and 1.89-2.16; P < 0.001), respectively. Among females, compared with those without constipation, there was a 1.86-fold higher risk of asthma in patients with constipation (aHR; 95\% CI, 1.77-1.96; P < 0.001). Among males, there was 1.92fold higher risk of asthma in patients with constipation (aHR; 95\% CI, 1.80-2.04; P < 0.001). Furthermore, constipated patients had a higher likelihood of asthma, regardless of comorbidities and medications.

In addition, Table 4 presents that analysis for risk of asthma in constipated patients with laxatives prescrip- 
tion. Compared to participants without constipation, the adjusted hazard ratio was 1.76 (95\% CI, 1.69-1.85; $\mathrm{P}<0.001), 2.15(95 \% \mathrm{CI}, 2.03-2.27 ; \mathrm{P}<0.001)$, and 2.29(95\% CI, 2.10-2.49; $\mathrm{P}<0.001)$ for $<3$ times, 3-12 times, and [?]12 times of laxatives prescription within one year, respectively. There appeared to be a dose-effect relationship between constipation severity and risk of asthma.The Kaplan-Meier curves are shown in Fig. 2. The cumulative incidence of asthma was significantly higher in constipated patients than in non-constipated patients, and the log-rank test for the comparison of cumulative incidence curves resulted in a $\mathrm{P}$-value of $<0.001$

\section{DISCUSSION}

In this study, people with constipation had a 1.91-fold higher risk for developing asthma than non-constipated individuals, regardless of age, gender, comorbidities or medications. As far as we know, this is the largest epidemiological research by using a nationwide longitudinal population-based dataset to expound the association between constipation and asthma. This relationship might be of pathophysiological and clinical importance. Our findings emphasize the considerably higher risk of asthma in individuals with constipation. Constipation might be influential in the development of asthma. Physicians should take care about the possibility of asthma in patients with constipation. Correspondingly, constipated patients should be informed of the probable risk of asthma and be offered with applicable management for asthma as required. Our findings further highlight the importance of maintaining fair bowel habits in order to avoid constipation, which could in turn mitigate risk of asthma.

We also observed that the risk of developing asthma was significantly increased in patients with hypertension, chronic obstructive pulmonary disease (COPD), chronic liver disease, and autoimmune disease. Most of these comorbidities, such as COPD or autoimmune disease, were connected to chronic inflammation, which could contribute to release of serum cytokines and activate T-cell response. In addition, we further extended to suggest that constipation severity is associated with the risk of asthma. There seemed to be a dose-dependent relationship between constipation and subsequent risk of asthma, which strengthened our hypothesis.

The pathophysiological mechanisms underlying the association between constipation and asthma remain ambiguous. Currently, there has been considerable studies conducted between intestinal microbiota and asthma.[17-21] The extended "hygiene hypothesis" discloses that the initial composition of the infant gut microflora plays a vital role in the development of atopic diseases.[22-24] Another research in United Kingdom, which analyzed the gut microbiome of the people with asthma, showed that there were abundant with Clostridiums spp. whereas Faecalibacterium prausnitzii and Bacteroides stercoris were depleted in individuals with asthma.[25] Similarly, some researches have suggested that alterations in the intestinal microflora would lead to constipation and constipation-related symptoms. $[5,7]$ In contrast to the healthy individuals, constipated patients had relatively higher amount of potentially pathogenic microbes, such as Clostridiums spp. and Pseudomonas aeruginosa, and relatively lower amount of Bacteroides spp., Bifidobacterium, and Lactobacillus.[26] These alternates in the intestinal surroundings could affect bowel motility by the active materials. Some studies exhibited that microbial-derived metabolites, mainly short-chain fatty acids (SCFAs), acted as pivotal drivers of T-cell subset activity and proliferation.[27] Furthermore, it has been disclosed that production of bowel microbial SCFAs could down-regulate proinflammatory reactions at the area of allergen insult.[28] Moreover, SCFAs might influence bowel motility via stimulating the contraction of colonic smooth muscles, thereby assisting to present or relieve constipation. $[29,30]$ By contrast, immune homeostasis would be devastated by proinflammatory dietary type, or "Westernized dietary style", which might be described as being low in fiber and high in fat, changing the intestinal microbiota, and leading to decrease SCFAs production. Hence, less fiber intake, such as fewer fresh fruits, in patients with constipation might be also pivotal role in developing atopy.[31, 32] Organizing the presently available corroboration suggests that the gut microbiota might be a key mechanistic part linking asthma and constipation. It is not known how constipation influence the configuration of the intestinal microflora and how applicable this condition is to asthma. Nevertheless, constipation appears to be the predisposing point for asthma. Further comprehensive metabolomic and metagenomic analyses of the gut microbiome in constipated individuals are warranted to clarify the potential mechanisms underlying these connections. 
The major advantages of this study were the relatively large sample size and long follow-up period. An integrated past history of used medical services was accessible for all cases. Therefore, there was slightest selection, information, and recall bias. As such, it was feasible to properly examine our hypothesis. However, there some potential limitations existed in our study. First, the NHIRD does not include data on covariates, such as social adversity, personal lifestyle, family history, laboratory data and environmental factors. Although we adjusted for several comorbidities and matched propensity scores, these unmeasured confounding factors could have influenced our results. Second, the diagnoses of asthma and constipation were totally dependent on the ICD-9 codes in the administrative dataset. We did not undergo a review of the patients' medical documents so that it was not probable to check the accuracy of diagnoses, and thus there might have some misclassifications existed. It is worth noting, however, that any misclassifications were more probably to be random, and connections were often underestimated rather than overestimated. Besides, clinical judgment might be different among clinicians, and so diagnoses would not have been consistent, which might have affected the validity. However, Taiwan's National Health Insurance administration monitors the accuracy of the claims data to prevent violations. Finally, it keeps unclear as to if the findings of our study may be extrapolated to other ethnic groups, as the majority of our subjects were Taiwanese. Further clinical research should include other nationalities and ethnicities to define the generalizability of the relationship observed herein.

\section{CONCLUSION}

In conclusion, constipated people had a 1.91-fold greater risk for asthma compared with those without constipation. Individuals with constipation should be alert to the elevated risk of developing asthma. Moreover, physicians should define the intestinal condition, including the gut microbiome, in patients with asthma. The precise pathophysiological association between constipation and asthma still needs further research.

\section{ACKNOWLEDGMENTS:}

None.

\section{CONFLICT OF INTEREST:}

There is no conflict of interest in this study.

\section{FUNDING:}

The authors have not declared a specific grant for this study from any funding agency in the public, commercial or not-for-profit sectors.

\section{AVAILABILITY OF DATA AND MATERIALS:}

The LHID is a subset of the NHIRD, a database of all medical claims in Taiwan's NHI system. The usage of NHIRD is limited to research purposes only. Only Taiwanese citizens who fulfill the requirements for conducting research projects are eligible to apply for access to the National Health Insurance Research Database (NHIRD). Applicants must follow the Personal Data Protection Act (https://law.moj.gov.tw/ENG/LawClass/LawAll.aspx?pcode=I0050021) and related regulations of the National Health Insurance Administration and NHRI (National Health Research Institutes), and an agreement must be signed by the applicant and his/her supervisor upon application submission. The datasets generated and analyzed during the current study are available from the authors on reasonable request.

\section{ETHICS APPROVAL AND CONSENT TO PARTICIPATE:}

This study was approved by the Institutional Review Board of Chung Shan Medical University Hospital (Approval number CS15134) in Taiwan. The requirement for written consent from study subjects was waived by the Institutional Review Board, as the LHID consists of de-identified secondary data.

\section{REFERENCES}


1. James, S.L., et al., Global, regional, and national incidence, prevalence, and years lived with disability for 354 diseases and injuries for 195 countries and territories, 1990-2017: a systematic analysis for the Global Burden of Disease Study 201\%. The Lancet, 2018.392 (10159): p. 1789-1858.

2. Chastek, B., et al., Economic Burden of Illness Among Patients with Severe Asthma in a Managed Care Setting. J Manag Care Spec Pharm, 2016. 22 (7): p. 848-61.

3. Leander, M., et al., Health-related quality of life predicts onset of asthma in a longitudinal population study. Respir Med, 2009.103 (2): p. 194-200.

4. Mugie, S.M., M.A. Benninga, and C. Di Lorenzo, Epidemiology of constipation in children and adults: a systematic review. Best Pract Res Clin Gastroenterol, 2011. 25 (1): p. 3-18.

5. Zhao, Y. and Y.B. Yu, Intestinal microbiota and chronic constipation. Springerplus, 2016. 5 (1): p. 1130.

6. Dimidi, E., et al., Mechanisms of Action of Probiotics and the Gastrointestinal Microbiota on Gut Motility and Constipation. Adv Nutr, 2017. 8 (3): p. 484-494.

7. Ohkusa, T., et al., Gut Microbiota and Chronic Constipation: A Review and Update. Front Med (Lausanne), 2019. 6 : p. 19.

8. Khalif, I.L., et al., Alterations in the colonic flora and intestinal permeability and evidence of immune activation in chronic constipation. Dig Liver Dis, 2005. 37 (11): p. 838-49.

9. Anand, S. and S.S. Mande, Diet, Microbiota and Gut-Lung Connection. Frontiers in Microbiology, 2018. 9 .

10. Frati, F., et al., The Role of the Microbiome in Asthma: The Gut-Lung Axis. International Journal of Molecular Sciences, 2018.20 (1).

11. Salameh, M., et al., The role of gut microbiota in atopic asthma and allergy, implications in the understanding of disease pathogenesis. Scandinavian Journal of Immunology, 2020. 91 (3).

12. Arrieta, M.-C., et al., A humanized microbiota mouse model of ovalbumin-induced lung inflammation. Gut Microbes, 2016. 7 (4): p. 342-352.

13. Ai, T., et al., Constipation might be associated with risk of allergic rhinitis: A nationwide populationbased cohort study. Plos One, 2020. 15 (10).

14. Huang, Y.C., et al., Influence of constipation on atopic dermatitis: A nationwide population-based cohort study in Taiwan.International Journal of Clinical Practice, 2020.

15. Zhu, L., et al., Structural changes in the gut microbiome of constipated patients. Physiological Genomics, 2014. 46 (18): p. 679-686.

16. Hsing, A.W. and J.P. Ioannidis, Nationwide Population Science: Lessons From the Taiwan National Health Insurance Research Database.JAMA Intern Med, 2015. 175 (9): p. 1527-9.

17. Ver Heul, A., J. Planer, and A.L. Kau, The Human Microbiota and Asthma. Clin Rev Allergy Immunol, 2019. 57 (3): p. 350-363.

18. Barcik, W., et al., The Role of Lung and Gut Microbiota in the Pathology of Asthma. Immunity, 2020. 52 (2): p. 241-255.

19. Chunxi, L., et al., The Gut Microbiota and Respiratory Diseases: New Evidence. J Immunol Res, 2020. 2020 : p. 2340670.

20. Salameh, M., et al., The role of gut microbiota in atopic asthma and allergy, implications in the understanding of disease pathogenesis. Scand J Immunol, 2020. 91 (3): p. e12855. 
21. Zhang, D., et al., The Cross-Talk Between Gut Microbiota and Lungs in Common Lung Diseases. Front Microbiol, 2020. 11 : p. 301.

22. Strachan, D.P., Hay fever, hygiene, and household size. BMJ (Clinical research ed.), 1989. 299 (6710): p. $1259-1260$.

23. Vael, C., et al., Denaturing gradient gel electrophoresis of neonatal intestinal microbiota in relation to the development of asthma. BMC Microbiol, 2011. 11 : p. 68.

24. Daley, D., The evolution of the hygiene hypothesis: the role of early-life exposures to viruses and microbes and their relationship to asthma and allergic diseases. Curr Opin Allergy Clin Immunol, 2014.14 (5): p. $390-6$.

25. Wang, Q., et al., A metagenome-wide association study of gut microbiota in asthma in UK adults. BMC Microbiol, 2018. 18 (1): p. 114.

26. Gerritsen, J., et al., Intestinal microbiota in human health and disease: the impact of probiotics. Genes Nutr, 2011. 6 (3): p. 209-40.

27. Smith, P.M., et al., The microbial metabolites, short-chain fatty acids, regulate colonic Treg cell homeostasis. Science, 2013.341 (6145): p. 569-73.

28. Lynch, S.V., Gut Microbiota and Allergic Disease. New Insights. Ann Am Thorac Soc, 2016. 13 Suppl 1 (Suppl 1): p. S51-4.

29. Dass, N.B., et al., The relationship between the effects of short-chain fatty acids on intestinal motility in vitro and GPR43 receptor activation. Neurogastroenterol Motil, 2007. 19 (1): p. 66-74.

30. Chu, J.R., et al., Prebiotic UG1601 mitigates constipation-related events in association with gut microbiota: A randomized placebo-controlled intervention study. World J Gastroenterol, 2019. 25 (40): p. 6129-6144.

31. Katsoulis, K., et al., Microbiota and asthma: Clinical implications. Respir Med, 2019. 146 : p. 28-35.

32. Spencer, S.P., G.K. Fragiadakis, and J.L. Sonnenburg, Pursuing Human-Relevant Gut MicrobiotaImmune Interactions. Immunity, 2019.51 (2): p. 225-239.

Table 1. Demographic characteristics of constipation group and non-constipation group

\begin{tabular}{lllll}
\hline & Before propensity score matched & Before propensity score matched & & After propensity \\
& Constipation $(\mathrm{N}=102467)$ & Non-constipation $(\mathrm{N}=102467)$ & ASD & Constipation $(\mathrm{N}=$ \\
Age & & & $<0.001$ & \\
$<20$ & $15501(15.1)$ & $15501(15.1)$ & & $13276(16.1)$ \\
$20-39$ & $31798(31.0)$ & $31798(31.0)$ & $25192(30.6)$ \\
$40-64$ & $30822(30.1)$ & $30822(30.1)$ & $24699(30.0)$ \\
65 & $24346(23.8)$ & $24346(23.8)$ & $<2.0$ & $19254(23.4)$ \\
Mean \pm SD & $44.1 \pm 22.7$ & $44.1 \pm 22.7$ & $<0.001$ & $43.6 \pm 22.8$ \\
Gender & & & & \\
Female & $68983(67.3)$ & $68983(67.3)$ & & $26061(31.6)$ \\
Male & $33484(32.7)$ & $33484(32.7)$ & 0.152 & $12212(14.8)$ \\
Hypertension & $18188(17.8)$ & $12651(12.3)$ & 0.083 & $3684(4.5)$ \\
Hyperlipidemia & $5500(5.4)$ & $3728(3.6)$ & 0.103 & $2109(2.6)$ \\
Chronic liver disease & $3829(3.7)$ & $2060(2.0)$ & 0.044 & $558(0.7)$ \\
Chronic kidney disease & $1042(1.0)$ & $638(0.6)$ & 0.146 & $5556(6.7)$ \\
Diabetes & $9368(9.1)$ & $5495(5.4)$ & 0.091 & $1374(1.7)$ \\
COPD & $2622(2.6)$ & $1337(1.3)$ & 0.043 & $571(0.7)$ \\
Autoimmune disease & $966(0.9)$ & $584(0.6)$ & &
\end{tabular}




\begin{tabular}{lllll}
\hline & Before propensity score matched & Before propensity score matched & & After propensity \\
\hline Corticosteroids & $25401(24.8)$ & $14964(14.6)$ & 0.258 & $14855(18.0)$ \\
Antihistamines & $71110(69.4)$ & $51862(50.6)$ & 0.391 & $51741(62.8)$ \\
\hline
\end{tabular}

ASD: Absolute standardized differences.

COPD: Chronic obstructive pulmonary disease.

Table 2. Cox proportional hazard model analysis for risk of asthma

\begin{tabular}{llllllll}
\hline & No. of asthma & PY & ID & Univariate & & Multivariate & \\
\hline & & & & HR $(95 \%$ C.I. $)$ & p value & HR (95\% C.I.) & p value \\
$\begin{array}{l}\text { Group } \\
\text { Non-constipation }\end{array}$ & 3740 & 662847 & 5.6 & 1 & & & \\
Constipation & 6622 & 610672 & 10.8 & $1.88(1.81-1.96)$ & $<0.001$ & $1.91(1.84-1.99)$ & $<0.001$ \\
Age & & & & & & & \\
$<20$ & 2250 & 249050 & 9.0 & 1 & & 1 & \\
$20-39$ & 1551 & 410253 & 3.8 & $0.40(0.37-0.42)$ & $<0.001$ & $0.37(0.35-0.40)$ & $<0.001$ \\
$40-64$ & 2721 & 377274 & 7.2 & $0.75(0.71-0.80)$ & $<0.001$ & $0.68(0.64-0.72)$ & $<0.001 ?$ ¿? \\
65 & 3840 & 236941 & 16.2 & $1.60(1.52-1.69)$ & $<0.001$ & $1.31(1.23-1.39)$ & $<0.001$ \\
Gender & & & & & & & \\
Female & 6252 & 903955 & 6.9 & 1 & & 1 & \\
Male & 4110 & 369563 & 11.1 & $1.57(1.51-1.63)$ & $<0.001$ & $1.17(1.12-1.22)$ & $<0.001$ \\
Hypertension & 2243 & 145764 & 15.4 & $1.98(1.89-2.07)$ & $<0.001$ & $1.22(1.15-1.29)$ & $<0.001$ \\
Hyperlipidemia & 517 & 43009 & 12.0 & $1.37(1.25-1.50)$ & $<0.001$ & $0.91(0.82-0.99)$ & 0.037 \\
Chronic liver disease & 334 & 26518 & 12.6 & $1.50(1.35-1.68)$ & $<0.001$ & $1.21(1.09-1.36)$ & $<0.001$ \\
Chronic kidney disease & 79 & 5131 & 15.4 & $1.65(1.32-2.06)$ & $<0.001$ & $1.06(0.85-1.32)$ & 0.624 \\
Diabetes & 836 & 61790 & 13.5 & $1.57(1.46-1.69)$ & $<0.001$ & $0.96(0.89-1.03)$ & 0.273 \\
COPD & 568 & 12955 & 43.8 & $5.16(4.74-5.61)$ & $<0.001$ & $3.21(2.94-3.50)$ & $<0.001$ \\
Autoimmune disease & 104 & 7533 & 13.8 & $1.63(1.35-1.98)$ & $<0.001$ & $1.42(1.17-1.73)$ & $<0.001$ \\
Corticosteroids & 1876 & 250654 & 7.5 & $0.93(0.89-0.98)$ & 0.007 & $0.80(0.76-0.84)$ & $<0.001$ \\
Antihistamines & 6212 & 915347 & 6.8 & $0.65(0.62-0.68)$ & $<0.001$ & $0.61(0.59-0.64)$ & $<0.001$ \\
\hline
\end{tabular}

ID: Incidence density (per 1000 person-years).

PY: person-years.

COPD: Chronic obstructive pulmonary disease.

Table 3. Subgroup analysis of the association between constipation and asthma development

\begin{tabular}{lll}
\hline & Constipation & Constipation \\
\hline Age & $\mathrm{N}$ & No. of asthma \\
$<20$ & & 1429 \\
$20-39$ & 13276 & 1019 \\
$40-64$ & 25192 & 1668 \\
65 & 24699 & 2506 \\
Gender & 19254 & 3980 \\
Female & &
\end{tabular}




\begin{tabular}{lll}
\hline & Constipation & Constipation \\
\hline Male & 26061 & 2642 \\
Hypertension & & \\
No & 70209 & 5193 \\
Yes & 12212 & 1429 \\
Hyperlipidemia & & \\
No & 78737 & 6308 \\
Yes & 3684 & 314 \\
Chronic liver disease & Chronic liver disease & \\
No & 80312 & 6401 \\
Yes & 2109 & 221 \\
Chronic kidney disease & Chronic kidney disease & \\
No & 81863 & 6571 \\
Yes & 558 & 51 \\
Diabetes & & \\
No & 76865 & 6084 \\
Yes & 5556 & 538 \\
Chronic obstructive pulmonary disease & Chronic obstructive pulmonary disease & Chronic obstructive pulmonary disease \\
No & 81047 & 6261 \\
Yes & 1374 & 361 \\
Autoimmune disease & Autoimmune disease & \\
No & 81850 & 6554 \\
Yes & 571 & 68 \\
Corticosteroids & & \\
No & 67566 & 5547 \\
Yes & 14855 & 1075 \\
Antihistamines & & \\
No & 30680 & 2836 \\
Yes & 51741 & 3786 \\
\hline & & \\
\hline & &
\end{tabular}

Table 4. Cox proportional hazard model analysis for risk of asthma in constipated patients with laxatives

\begin{tabular}{|c|c|c|c|c|c|}
\hline & $\mathrm{N}$ & No. of asthma & Univariate & & Multivariate \\
\hline & & & HR (95\% C.I.) & $\mathrm{p}$ value & HR $(95 \%$ C.I \\
\hline \multicolumn{6}{|l|}{ Group } \\
\hline Non-constipation & 82421 & 3740 & 1 & & 1 \\
\hline Constipation with Laxatives prescription $<3$ times & 55070 & 3929 & $1.59(1.52-1.66)$ & $<0.001$ & $1.76(1.69-1.8$ \\
\hline Constipation with Laxatives prescription $=3-12$ times & 21332 & 2000 & $2.37(2.24-2.50)$ & $<0.001$ & $2.15(2.03-2.2$ \\
\hline Constipation with Laxatives prescription [?]12 times & 6019 & 693 & $3.39(3.13-3.68)$ & $<0.001$ & $2.29(2.10-2.4$ \\
\hline
\end{tabular}

+Adjusted for age, gender, hypertension, hyperlipidemia, chronic liver disease, chronic kidney disease, diabetes, COPD, autoimmune disease, corticosteroids, and antihistamines. 


\begin{tabular}{|c|c|}
\hline $\begin{array}{l}\text { Longitudinal health insurance database } \\
\qquad N=1000000\end{array}$ & 7 \\
\hline $\begin{array}{l}\text { Newly diagnosed constipation (ICD-9- } \\
C M=564.0 \text { ) and at leas } \geq 3 \text { OPD visits } \\
\text { or one admission from } 2000 \text { to } 2012 \\
\qquad N=122359\end{array}$ & $\begin{array}{l}\text { Never diagnosed constipation (ICD-9. } \\
\begin{array}{c}\mathrm{CM}=564.0 \text { ) from } 1999 \text { to } 2013 \\
\mathrm{~N}=708497\end{array}\end{array}$ \\
\hline \multirow{2}{*}{$\begin{array}{c}\text { Exclusion of asthma (ICD-9-CM=493) } \\
\text { diagonosis before index date } \\
\mathrm{N}=103952\end{array}$} & $\begin{array}{l}\text { Matched } 1: 1 \text { by age and gender } \\
\qquad N=102467\end{array}$ \\
\hline & \\
\hline $\begin{array}{l}\text { Matched } 1: 1 \text { by age and gender } \\
\qquad N=102467\end{array}$ & \\
\hline $\begin{array}{c}\text { Constipation } \\
\text { Propensity score matched 1: } 1 \text { by age, } \\
\text { gender, hypertension, hyperlipidemia, } \\
\text { chronic liver disease, chronic kidney } \\
\text { disease, diabetes, COPD, autoimmune } \\
\text { disease, corticosteroids, and } \\
\text { antihistamines } \\
\mathrm{N}=\$ 2421\end{array}$ & $\begin{array}{l}\text { Non-con stipation } \\
\text { Propensity score matched } 1: 1 \text { by age, } \\
\text { gender, hypertension, hyperlipidemia, } \\
\text { chronic liver disease, chronic ki dney } \\
\text { disease, diabetes, COPD, autoimmune } \\
\text { disease, corticosteroids, and } \\
\text { antihistamines } \\
\mathrm{N}=\$ 2421\end{array}$ \\
\hline
\end{tabular}

Figure 1. Flowchart of enrolment of constipation and non-constipation groups. 


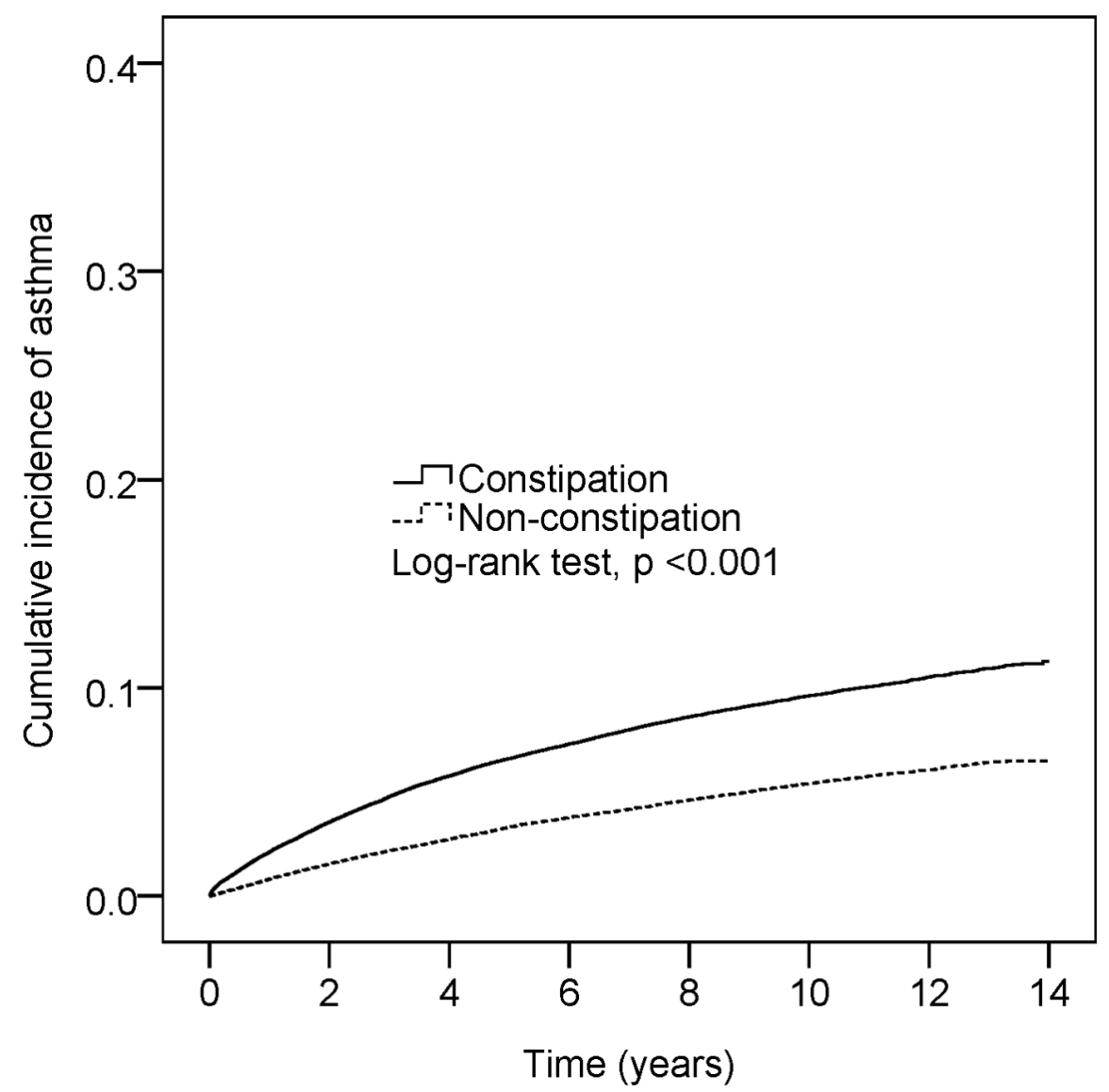

Figure 2. Kaplan-Meier curve of cumulative incidence proportion of asthma in constipation and nonconstipation groups. 


\begin{tabular}{|c|c|}
\hline $\begin{array}{l}\text { Longitudinal health insurance database } \\
\qquad N=1000000\end{array}$ & \\
\hline $\begin{array}{l}\text { Newly diagnosed constipation (ICD-9- } \\
C M=564.0 \text { ) and at least } 23 \text { OPD vists } \\
\text { or one admission from } 2000 \text { to } 2012 \\
\qquad N=122359\end{array}$ & $\begin{array}{l}\text { Never diagnosed constipation (ICD-9- } \\
\begin{array}{c}\mathrm{CM}=564.0 \text { ) from } 1999 \text { to } 2013 \\
\mathrm{~N}=708497\end{array}\end{array}$ \\
\hline \multirow{2}{*}{$\begin{array}{c}\text { Exclusion of asthma (ICD-9-CM=493) } \\
\text { diagonosis before index date } \\
\mathrm{N}=103952\end{array}$} & $\begin{array}{l}\text { Matched } 1: 1 \text { by age and gender } \\
\qquad N=102467\end{array}$ \\
\hline & \\
\hline $\begin{array}{l}\text { Matched } 1: 1 \text { by age and gender } \\
\qquad N=102467\end{array}$ & \\
\hline $\begin{array}{l}\text { Constipation } \\
\text { Propensity score matched 1: } 1 \text { by age, } \\
\text { gender, hypertension, hyperlipidemia, } \\
\text { chronic liver disease, chronic kidney } \\
\text { disease, diabetes, COPD, autoimmune } \\
\text { disease, corticosteroids, and } \\
\text { antihistamines } \\
\mathrm{N}=\$ 2421\end{array}$ & $\begin{array}{l}\text { Non-constipation } \\
\text { Propensity score matched } 1: 1 \text { by age, } \\
\text { gender, hypertension, hyperlipidemia, } \\
\text { chronic liver disease, chronic kidney } \\
\text { disease, diabetes, COPD, autoimmune } \\
\text { disease, corticosteroids, and } \\
\text { antihistamines } \\
\mathrm{N}=\$ 2421\end{array}$ \\
\hline
\end{tabular}




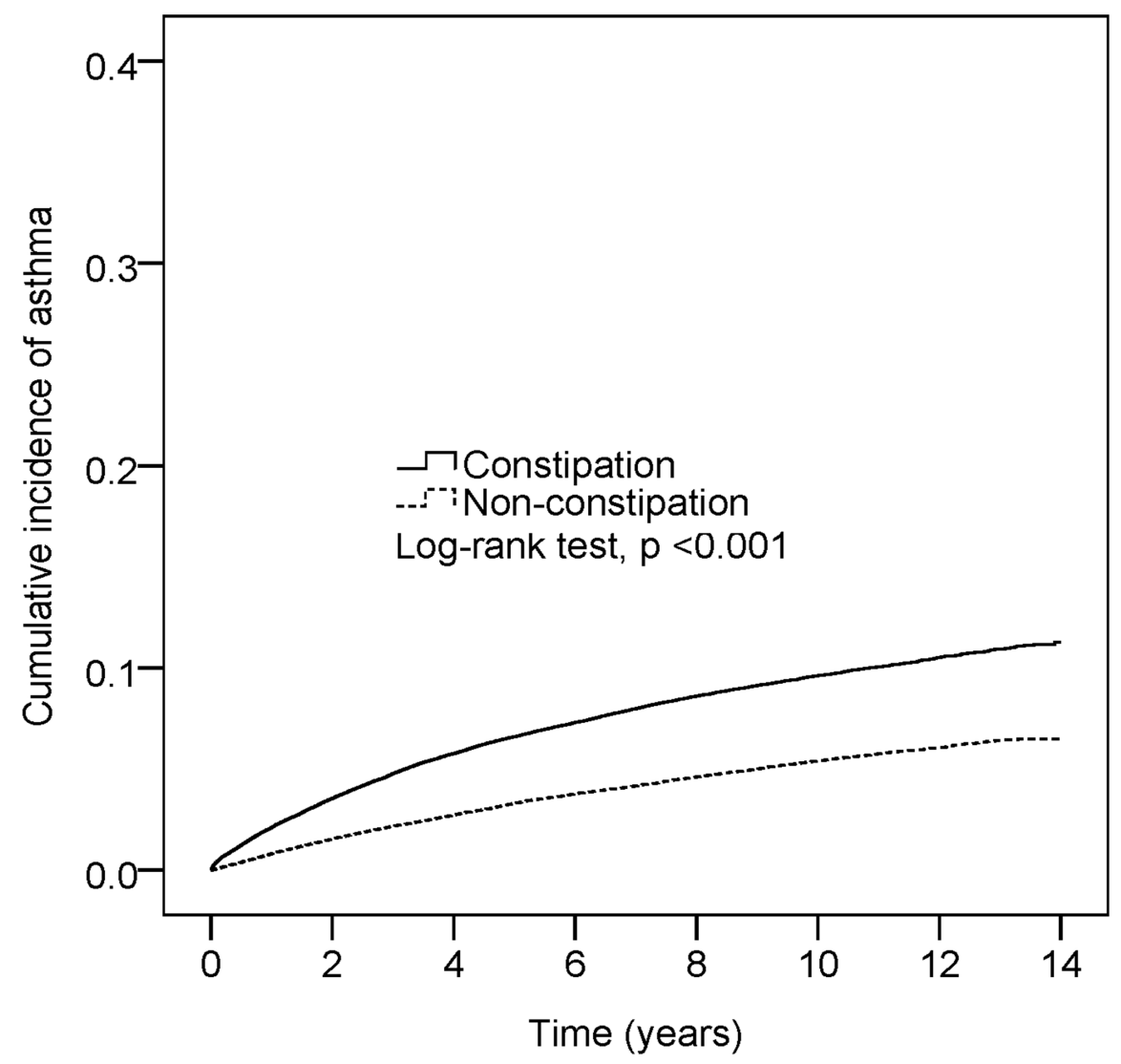

\section{Hosted file}

Table1.pdf available at https://authorea.com/users/331375/articles/504435-the-influence-ofconstipation-on-asthma-a-real-world-population-based-cohort-study

\section{Hosted file}

Table2.pdf available at https://authorea.com/users/331375/articles/504435-the-influence-ofconstipation-on-asthma-a-real-world-population-based-cohort-study

\section{Hosted file}

Table3.pdf available at https://authorea.com/users/331375/articles/504435-the-influence-ofconstipation-on-asthma-a-real-world-population-based-cohort-study

\section{Hosted file}

Table4.pdf available at https://authorea.com/users/331375/articles/504435-the-influence-ofconstipation-on-asthma-a-real-world-population-based-cohort-study 